\title{
MICROALGAS CHLOROPHYTAS E VARIÁVEIS LIMNOLÓGICAS DE UMA MICROBACIA URBANA - CUIABÁ-MATO GROSSO/BRASIL
}

\author{
R. L. COSTA*, F. P. SCHULTS, O. C. NASCIMENTO \\ Universidade Federal de Mato Grosso \\ regiluiza.bio@gmail.com*
}

Submetido em 13/04/2015 e aceito em 25/02/2018

DOI: $10.15628 /$ holos.2020.2969

\begin{abstract}
RESUMO
Avaliações ambientais quando associam bioindicadores e variáveis limnológicas possuem maior precisão em diagnosticar a qualidade explicar ações de causa e efeito de um ecossistema. Este trabalho tem o objetivo de medir variáveis limnológicas e realizar o levantamento de microalgas Chlorophytas, na Lagoa Paiaguás- Cuiabá, Mato Grosso, afim de caracterizar a qualidade ambiental desse ecossistema aquático urbano, visando a geração de informações que possam vir a servir como subsídios a gestão de recursos hídricos. As análises indicaram uma água com
\end{abstract}

\begin{abstract}
tendência a neutralidade, temperaturas altas e variações de nutrientes. Qualitativamente as Chlorophytas apresentaram 5 gêneros distribuídos em 3 famílias no período de estiagem e 14 gêneros distribuídos em 7 famílias no período de cheia. As variáveis limnológicas podem influenciar a composição qualitativa das microalgas, pois em todos os parâmetros analisados houve relação direta ou indireta com o metabolismo aquático da Lagoa Paiaguás.
\end{abstract}

PALAVRAS-CHAVE: Parâmetros Ambientais, Bioindicadores, Recursos Hídricos.

\section{CHLOROPHYTAS MICROALGAE AND LIMNOLOGICAL VARIABLES IN THE URBAN MICRO-BASIN- CUIABÁ- MATO GROSSO/ BRAZIL}

\begin{abstract}
Environmental Parameters when associated bioindicators and limnological variables have greater accuracy in diagnosing the quality making it possible to detect and explain action of cause and effect an ecosystem. The aim of this work is to measure limnological variables and carry out the survey of micro algae Chlorophytas, in the Lagoa Paiaguás Cuiaba, Mato Grosso in order to characterize the environmental quality of urban aquatic ecosystem, aiming to generate

management of water resources. The analyzes indicated a tendency to neutrality with water, high temperatures and variations of nutrients. Qualitatively showed Chlorophytas 5 genus distributed in 3 families in the dry season, end 14 genus distributed in 7 families in the rainy season. Limnological variables likely to influence the qualitative composition of microalgae, because in all parameters analyzed were directly or indirectly involved with the aquatic metabolism in the Lagoa Paiaguás.
\end{abstract} information that may serve as subsidies the

KEYWORDS: Environmental Parameters, Bioindicators, Water Resources. 


\section{INTRODUÇÃO}

O monitoramento de qualidade das águas é um dos mais importantes instrumentos da gestão ambiental. Consistindo, no acompanhamento sistemático dos aspectos qualitativos e quantitativos das águas, visando à produção de informações com linguagem que garanta acessibilidade tanto para a comunidade científica, público em geral e, principalmente, às diversas instâncias decisórias (SEMA, 2007).

A adequada gestão dos recursos hídricos é tema recorrente na atualidade, sendo dessa forma necessário o monitoramento das bacias hidrográficas, tanto em áreas rurais como em áreas urbanas, uma vez que a disponibilidade hídrica, em especial para fins nobres como o abastecimento humano tem sofrido restrições significativas em função do comprometimento de seus aspectos de qualidade e quantidade (CUNHA e CALIJURI, 2010).

Dessa forma é imprescindível e cada vez mais necessário realizar o acompanhamento da qualidade da água afim de verificar a dinâmica ambiental e também aspectos referentes a sua disponibilidade tanto para a sociedade como para a biota aquática. Marques, et al., (2002) ressaltam que as avaliações ambientais quando associam bioindicadores e variáveis físico-químicas possuem maior precisão em diagnóstico de qualidade, através dessas torna-se possível, detectar e explicar ações de causa e efeito de um ecossistema, conhecer seu funcionamento a estrutura de uma comunidade em relação a sua característica natural, e as respostas e adaptações tanto de natureza física, química e biológica em relação aos efeitos antrópicos e usos esperados ou inesperados.

Dentre os micro-organismos aquáticos as microalgas são representativas como bases de cadeias ecológicas nos ambientes onde ocorrem, desempenhando um papel importante, sendo produtoras primárias de alimentos para o restante da magnificação trófica. Apesar da simplicidade morfológica e fisiológica elas podem ser usadas para diversos fins dentre os quais avaliações de qualidade ambiental, pois o uso de indicadores biológicos permite diagnosticar as condições limnológicas das áreas investigadas do ponto de vista da sua biodiversidade, uma vez que a estrutura da comunidade fica conhecida (JUNQUEIRA et al., 2000).

Diante desse cenário este trabalho tem o objetivo de medir variáveis limnológicas e realizar o levantamento de microalgas Chlorophytas, na Lagoa Paiaguás- Cuiabá, Mato Grosso, afim de caracterizar a qualidade ambiental desse ecossistema aquático urbano, tendo em vista a geração de informações que possam vir a servir como subsídios à para a gestão de recursos hídricos.

\section{METODOLOGIA}

Entre o Bairro Paiaguás e o Centro Político Administrativo do perímetro urbano da cidade de Cuiabá Estado de Mato Grosso, em frente à Assembleia Legislativa encontra-se a Lagoa Paiaguás. Composta de várias nascentes localizadas nas suas proximidades, sendo formadora do córrego Quarta Feira, que deságua no Ribeirão do Lipa. Possui 33 hectares de extensão e 
aproximadamente 7 metros de profundidade, no período chuvoso (SEMA, 2007). Nessa lagoa foram escolhidos 05 pontos de coleta conforme apresentado na Figura 1, para assim conseguir uma melhor representatividade da qualidade atual, desse corpo hídrico, sendo esses:

- Ponto 1 - Entrada da lagoa: Coordenadas Geográficas: $15^{\circ} 33^{\prime} 52,2^{\prime \prime} \mathrm{S} 56^{\circ} 04^{\prime} 39,4^{\prime \prime}$ W;

- Ponto 2 - Centro da lagoa: Coordenadas Geográficas: $15^{\circ} 34^{\prime} 04,9^{\prime \prime}$ S 56 04'47,7" W;

- $\quad$ Ponto 3- Margem esquerda lançamento de efluente: Coordenadas Geográficas: $15^{\circ} 34^{\prime} 04,9^{\prime \prime} \mathrm{S} 56^{\circ} 04^{\prime} 43,0^{\prime \prime} \mathrm{W}$;

- Ponto 4 - Vertedouro: Coordenadas Geográficas: $15^{\circ} 34^{\prime} 04,3^{\prime \prime}$ S 5604'47,9” W;

- Ponto 5 - Margem direita: Coordenadas Geográficas: $15^{\circ} 33^{\prime} 59,8^{\prime \prime}$ S 56 04' 48,0" W.

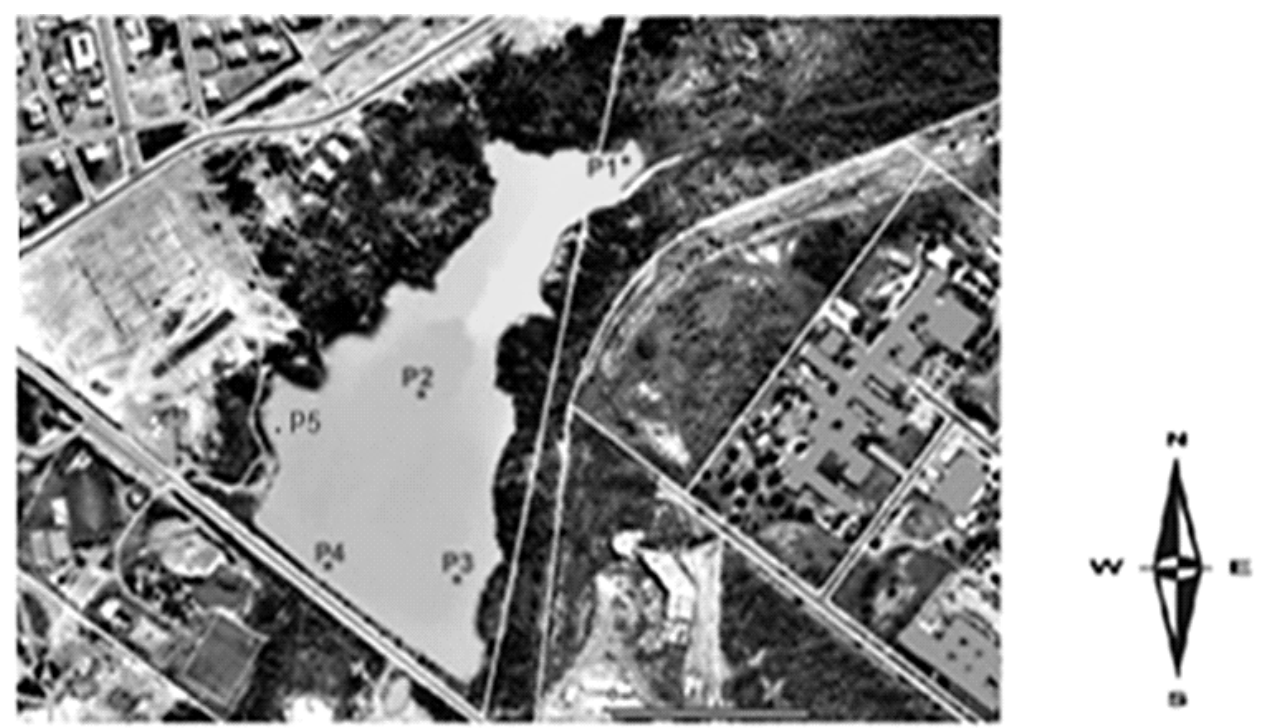

Figura 1- Pontos de amostragens da Lagoa Paiaguás- Cuiabá Mato Grosso Fonte: SEMA (2007) adaptado por Costa (2011).

A coleta de água foi em dois períodos distintos, cheia (fevereiro/2011) e seca (agosto/2011). A metodologia da coleta da água para as análises físico-químicas foi de acordo com o Guia de Coleta e Preservação de Amostras de Água da CETESB (1988), a amostragem biológica foi executada com uma rede de fitoplâncton de malha de $20 \mu \mathrm{m}$, conforme Bicudo e Bicudo (1970). Armazenou-se o material coletado em frasco de polietileno de capacidade de $250 \mathrm{ml}$ e preservado com solução de Transeau (BICUDO e BICUDO ,1970), logo em seguida identificaram-se numericamente.

As amostras das microalgas foram encaminhadas para o Laboratório de Botânica e Ecologia, do Instituto de Biociências da Universidade Federal de Mato Grosso (IB- UFMT). Para cada amostra analisou-se 10 lâminas, a visualização das espécies efetuou-se com auxílio de microscópio óptico com câmera acoplada em ampliação total de 40X e uso de ocular de medição com escala de $\mu \mathrm{m}$, nas identificações taxonômicas utilizaram trabalhos científicos como os de Bicudo e Bicudo (1970), Bourelly (1972), Bicudo e Menezes (2006), Gentil (2007), Bortolini et al., (2010 a, b), Hentschke e Torgan (2010), Souza e Melo (2011). 
Os procedimentos utilizados para as análises físico-químicas realizadas na Secretaria de Estado de Meio Ambiente (SEMA) Coordenadoria de Monitoramento da Qualidade Ambiental vão de acordo com o proposto pela APHA - AMERICAN PUPLIC HEALTH ASSOCIATION, $21^{\text {TH }}$ Edition 2005, conforme Tabela 1 onde nessa se encontram descritos os métodos utilizados.

Tabela 1: Variáveis analisadas e metodologia adotada para a Lagoa Paiaguás.

\begin{tabular}{l|l}
\hline \multicolumn{1}{c|}{ Variáveis } & \multicolumn{1}{c}{ Métodos } \\
\hline $\mathrm{pH}$ & Eletrométrico \\
Demanda Bioquímica de Oxigênio & Eletrométrico - Diluição e Incubação 20C por 5 dias \\
Temperatura da água & Eletrométrico \\
Condutividade elétrica & Eletrométrico \\
Oxigênio dissolvido & Eletrométrico \\
Turbidez & Turbidimetro/Nefelométrico \\
Nitrogênio-Nitrato & Espectrofotométrico \\
Fósforo total & Espectrofotométrico \\
\hline
\end{tabular}

Fonte: APHA(2005)

Executou-se uma comparação dos resultados obtidos no período de estiagem em todos os pontos amostrais com a Resolução CONAMA 357/2005 que dispõe sobre a classificação dos corpos de água e diretrizes ambientais para o seu enquadramento e dá outras providências considerando a lagoa com um corpo hídrico classe II, visto que os corpos hídricos do Estado de Mato Grosso ainda não possuem enquadramento (ALVES et al., 2009).

\section{RESULTADOS E DISCUSSÕES}

Houve uma ampla distinção qualitativa das espécies de microalgas entre as variações sazonais, porém a profusão no período chuvoso foi pouco representativa comparada com os dados da seca, onde se verificou a presença de 5 gêneros de espécies distribuídos em 3 famílias, no período de cheia caracterizadas na Tabela 2.

Tabela 2: Chlorophytas encontradas na amostragem em fevereiro de 2011na Lagoa Paiaguás em Cuiabá-MT.

\begin{tabular}{|c|c|c|}
\hline PONTOS & ESPÉCIES & FAMÍLIAS \\
\hline Ponto 1 & $\begin{array}{l}\text { Ankistrodesmus densus Corda } 1838 \\
\text { Scenedesmus acuminatus Meyen } 1829 \\
\text { Eudorina sp. }\end{array}$ & $\begin{array}{c}\text { Oocystaceae } \\
\text { Scenedesmaceae }\end{array}$ \\
\hline Ponto 2 & $\begin{array}{l}\text { Ankistrodesmus fusiformes Corda } 1838 \\
\text { Ankistrodesmus spp. }\end{array}$ & Oocystaceae \\
\hline Ponto3 & $\begin{array}{l}\text { Ankistrodesmus densus Corda } 1838 \\
\text { Ankistrodesmus fusiformes Corda } 1838 \\
\text { Scenedesmus baculiformis Meyen } 1829 \\
\text { Staurastrum sebaldii Meyen ex Ralfs } 1848\end{array}$ & $\begin{array}{c}\text { Oocystaceae } \\
\text { Scenedesmaceae }\end{array}$ \\
\hline Ponto 4 & Cosmarium quadrum Corda ex Ralfs 1848 & Desmidiaceae \\
\hline
\end{tabular}




\begin{tabular}{l|l|l}
\hline Ponto 5 & Ankistrodesmus spp. & Oocystaceae \\
\hline
\end{tabular}

No período de seca, houve mudança gradativa das espécies comparada com o período de cheia conforme mostrado na tabela 3. Nesse período foram encontrados 14 gêneros de microalgas distribuídos em 7 famílias.

Tabela 3: Chlorophytas encontradas na amostragem em Agosto de 2011 na Lagoa Paiaguás em Cuiabá-MT.

\begin{tabular}{|c|c|c|}
\hline PONTOS & ESPÉCIES & FAMÍLIAS \\
\hline Ponto 1 & $\begin{array}{l}\text { Akistrodesmus espiralis Corda } 1838 \\
\text { Ankistrodesmus densus Corda } 1838 \\
\text { Ankistrodesmus falcatus Corda } 1838 \\
\text { Ankistrodesmus fusiformes Corda } 1838 \\
\text { Ankistrodesmus spp. } \\
\text { Coelastrum prosbocidium Nägeli } 1849 \\
\text { Coelastrum microporum Nägeli } 1849 \\
\text { Coelastrum reticulatum Nägeli } 1849 \\
\text { Cosmarium quadrum Corda ex Ralfs } 1848 \\
\text { Crucigenia quadrata Morren } 1830 \\
\text { Dictyosphaerium pulchellum Nägeli } 1849 \\
\text { Eudorina spp. } \\
\text { Scenedesmus acuminatus Meyen } 1829 \\
\text { Scenedesmus crassus Meyen } 1829 \\
\text { Tetraedrum minimum Kützing } 1845\end{array}$ & $\begin{array}{c}\text { Oocystaceae } \\
\text { Scenedesmaceae } \\
\text { Desmidiaceae } \\
\text { Dictyosphaeriaceae } \\
\text { Chlorococcaceae }\end{array}$ \\
\hline Ponto 2 & $\begin{array}{l}\text { Ankistrodemus spp. } \\
\text { Ankistrodesmus fusiformes Corda } 1838 \\
\text { Closterium sp. } \\
\text { Coelastrum reticulatum Nägeli } 1849 \\
\text { Crucigenia quadrata Morren } 1830 \\
\text { Desmodesmus sp. } \\
\text { Eudorina spp. } \\
\text { Neproclamys sp. } \\
\text { Oocystis sp. } \\
\text { Staurastrum spp. } \\
\text { Tetraedrum minimum Kützing } 1845\end{array}$ & $\begin{array}{c}\text { Oocystaceae } \\
\text { Desmidiaceae } \\
\text { Scenedesmaceae } \\
\text { Chlorococcaceae }\end{array}$ \\
\hline Ponto 3 & $\begin{array}{l}\text { Ankistrodemus densus Corda } 1838 \\
\text { Ankistrodemus spp. } \\
\text { Coelastrum microporum Nägeli } 1849 \\
\text { Coelastrum reticulatum Nägeli } 1849 \\
\text { Crucigenia quadrata Morren } 1830 \\
\text { Desmodesmus ssp. } \\
\text { Scenedesmus spp. }\end{array}$ & $\begin{array}{c}\text { Oocystaceae } \\
\text { Scenedesmaceae }\end{array}$ \\
\hline
\end{tabular}


Continuação...

\begin{tabular}{|c|c|c|}
\hline PONTOS & ESPÉCIES & FAMÍLIAS \\
\hline Ponto 4 & $\begin{array}{l}\text { Ankistrodesmus densus Corda } 1838 \\
\text { Ankistrodesmus espiralis Corda } 1838 \\
\text { Ankistrodesmus falcatus Corda } 1838 \\
\text { Coelastrum proboscideum Nägeli } 1849 \\
\text { Coelastrum reticulatum Nägeli } 1849 \\
\text { Coleochaete sp. } \\
\text { Cosmarium quadrum Corda ex Ralfs } 1848 \\
\text { Crucigenia quadrata Morren } 1830 \\
\text { Dyctyosphaerium spp. } \\
\text { Eudorina spp. } \\
\text { Oedogonium spp. } \\
\text { Scenedesmus acuminatus Meyen } 1829 \\
\text { Scenedesmus baculiformes Meyen } 1829 \\
\text { Scenedesmuss spp. }\end{array}$ & $\begin{array}{c}\text { Oocystaceae } \\
\text { Scenedesmaceae } \\
\text { Desmidiaceae } \\
\text { Dictyosphaeriaceae } \\
\text { Chaetophoraceae }\end{array}$ \\
\hline Ponto 5 & $\begin{array}{l}\text { Ankistrodesmus densus Corda } 1838 \\
\text { Ankistrodesmus espiralis Corda } 1838 \\
\text { Closterium sp. } \\
\text { Coelastrum microporum Nägeli } 1849 \\
\text { Coelastrum reticulatum Nägeli } 1849 \\
\text { Coelastrum spp. } \\
\text { Crucigenia crucífera Morren } 1830 \\
\text { Eudorina spp. } \\
\text { Scenedesmus acuminatus Meyen } 1829 \\
\text { Scenedesmus arcuatus Meyen } 1829 \\
\text { Scenedesmus spp. } \\
\text { Staurastrum sebaldii Meyen ex Ralfs } 1848 \\
\text { Tetraedrum minimum Kützing } 1845\end{array}$ & $\begin{array}{c}\text { Oocystaceae } \\
\text { Desmidiaceae } \\
\text { Scenedesmaceae } \\
\text { Chlorococcaceae }\end{array}$ \\
\hline
\end{tabular}

Esse fato pode ter ocorrido devido à menor quantidade de chuvas no período de estiagem, podendo ter reduzido a quantidade de água na lagoa, havendo assim uma maior concentração de cargas de compostos orgânicos tais como efluentes domésticos e biomassa de vegetais em decomposição aumentando os nutrientes que favorecem a dinâmica ambiental das microalgas.

Dentre os gêneros de microalgas presentes na Lagoa Paiaguás o que provavelmente apresentou maior resistência as alterações ambientais e sazonais foi Ankistrodesmus (família Oocystaceae), pois esteve presente em 4 dos 5 pontos amostrais no período de cheia ( fevereiro) 
e nos 5 pontos amostrais do período da seca (agosto) .Como ressalta Calijuri (1988), a comunidade fitoplanctônica é constituída por grande número de espécies que possuem formas e estratégias de sobrevivência distintas, estando dessa forma capazes de adaptar suas funções metabólicas às diferentes mudanças ambientais e hidrológicas.

As variaveis que apresentaram valores abaixo dos limites considerados da Resolução CONAMA 357/2005 foram : oxigênio dissolvido no ponto1 (cheia) com valor de 4,4 mg/L menor que o estabelecido pela resolução, potençial Hidrogeniônico $(\mathrm{pH})$ nos 5 pontos amostrais da cheia com valor acima de 9,0, e fósforo total com valor maior que o máximo do enquadramento em todos os pontos de amostragem.

A temperatura da água teve pouca alteração de um período para o outro, porém mesmo estando entre $28,44^{\circ} \mathrm{C}$ e $30,30^{\circ} \mathrm{C}$ são valores altos (Figura 2). Os maiores valores foram registrados no período chuvoso indo de $28,7{ }^{\circ} \mathrm{C}$ (ponto 1 ) à $30,3{ }^{\circ} \mathrm{C}$ (ponto 4). No período de seca os pontos $1,2,3$ e 4 apresentaram valores em torno de $28,0^{\circ} \mathrm{C}$ e o ponto 5 teve o maior valor de $29,04^{\circ} \mathrm{C}$.

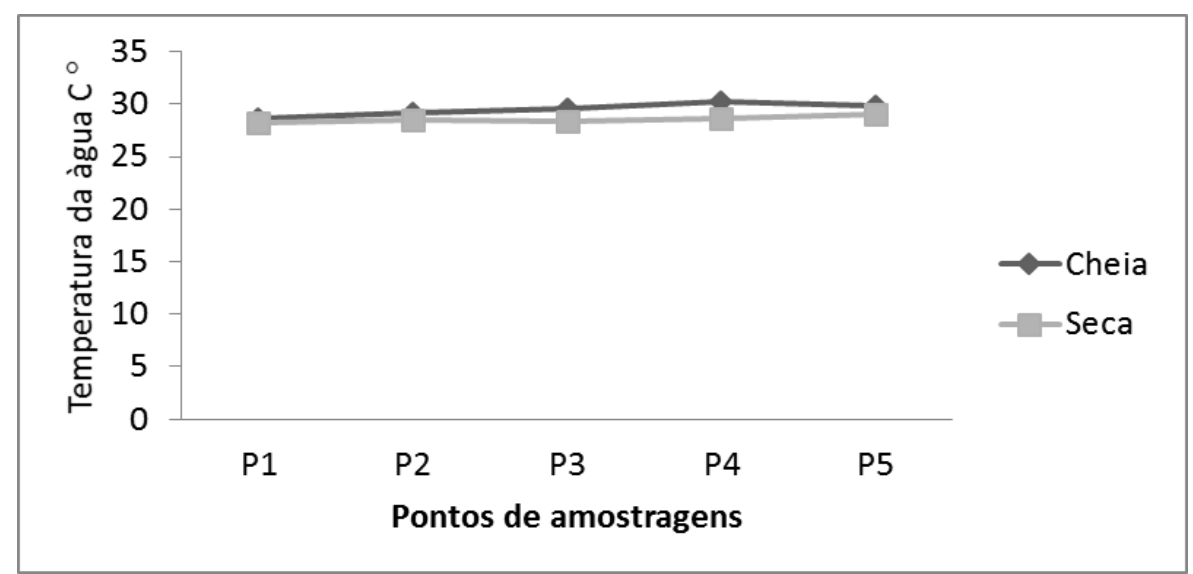

Figura 2: Comparação dos valores de Temperatura da Água, nos pontos de amostragens, nos períodos de Seca e Cheia na Lagoa Paiaguás em 2011.

A temperatura da água influi de maneira fundamental nos ciclos físicos e químicos dos lagos e reservatórios e, portanto, nos processos de produção e respiração que ocorrem nesses ecossistemas aquáticos. A elevação anormal da temperatura em um corpo hídrico geralmente é provocada por despejos industriais. (SEMA, 2011).

A Figura 3 expõem a variação do $\mathrm{pH}$, sendo que os valores de 7,27 a 9,87 indicaram tendência de neutro a alcalino. Conforme a SEMA (2011) este parâmetro pode definir o caráter ácido, básico ou neutro de uma solução e deve ser considerado, pois a microflorula aquática geralmente está adaptada às condições de neutralidade o que de fato influencia a fisiologia desses organismos. 


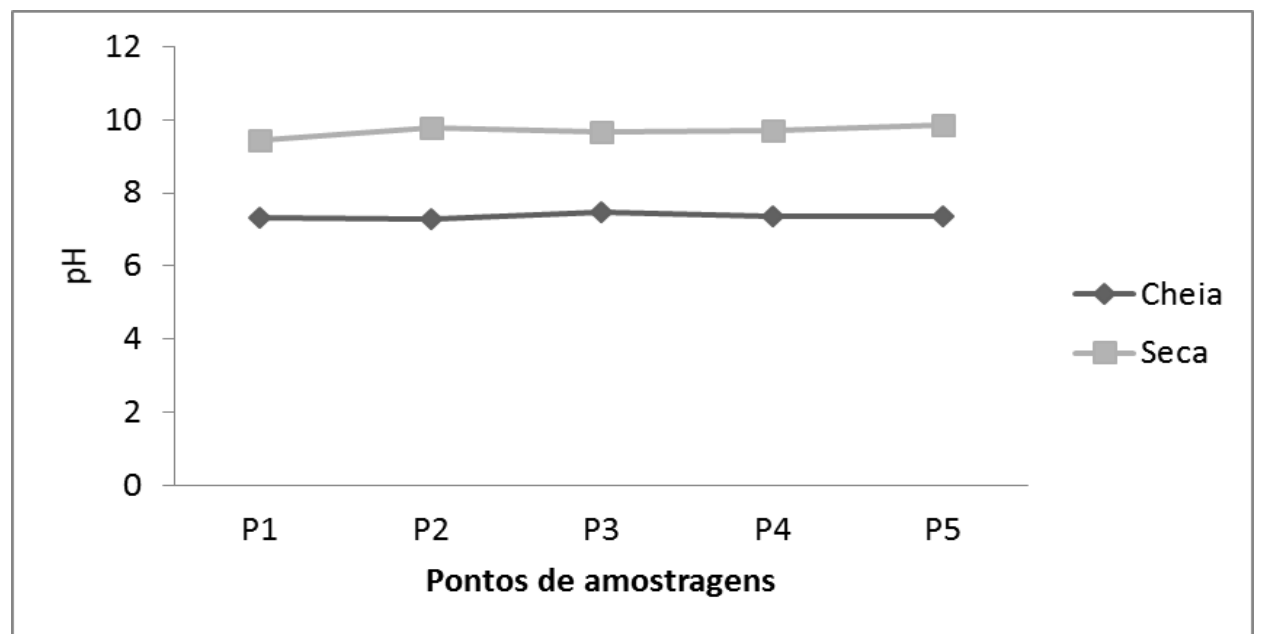

Figura 3: Comparação dos valores de potencial Hidrogeniônico, nos pontos de amostragens, nos períodos de Seca e Cheia na Lagoa Paiaguás em 2011.

Durante o período de seca a Lagoa Paiaguás apresentou material orgânico suspenso e em decomposição sendo a maioria oriunda da macrófita exótica Nelumbo sp. que alcançou grande reprodução nos pontos 2 e 3 . Esse fator pode ter contribuído para a diferença na quantidade de gêneros de microalgas Chlorophytas, pois no primeiro período de coleta (cheia) foram registrados 5 gêneros e na seca ocorreram 14 gêneros.

A caracterização das variações da Condutividade Elétrica da água, na Lagoa Paiaguás, está descrita na Figura 4 onde as maiores taxas de valores estiveram no período de seca estando o ponto $1 \mathrm{com} 148 \mu \mathrm{s} / \mathrm{cm}$ e os demais 4 pontos (ponto 2,3,4 e 5), tiveram $147 \mu \mathrm{s} / \mathrm{cm}$. No período da cheia foram registrados os menores valores no ponto $1,76 \mu \mathrm{s} / \mathrm{cm}$ e o valor mais alto no ponto 3 , $80 \mu \mathrm{s} / \mathrm{cm}$.

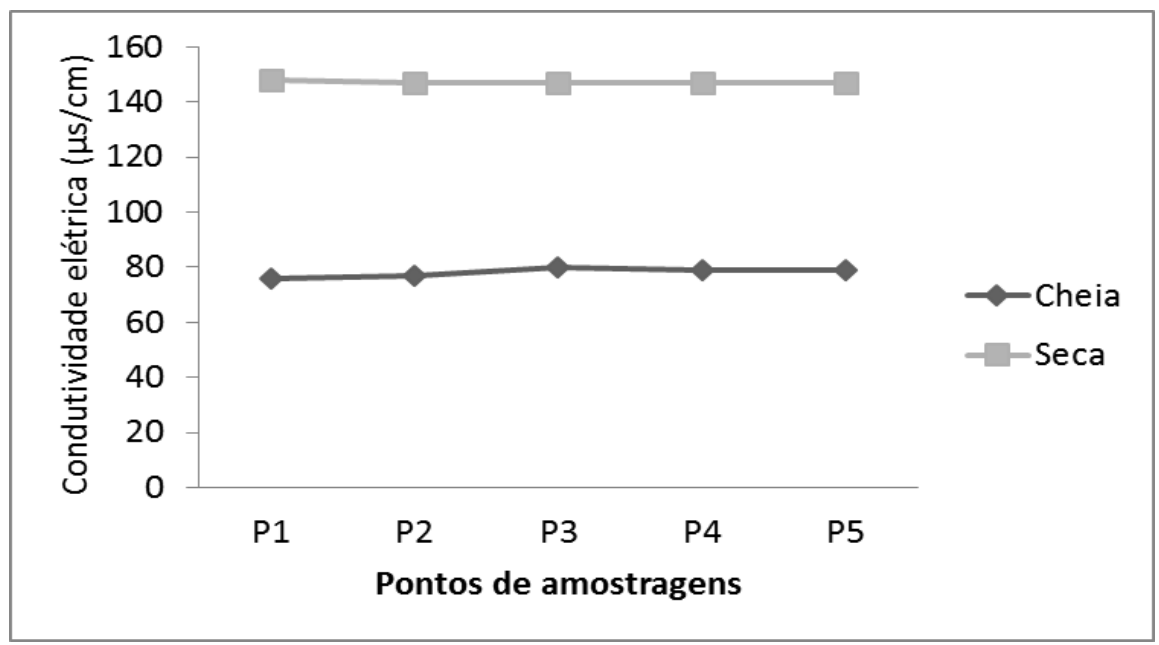

Figura 4: Comparação dos valores de Condutividade Elétrica da Água, nos pontos de amostragens, nos períodos de Seca e Cheia na Lagoa Paiaguás em 2011.

A condutividade depende das concentrações iônicas e da temperatura, fornecendo uma boa indicação das modificações na composição da água, especialmente na sua concentração mineral, mas não fornece nenhuma indicação das quantidades relativas dos vários componentes; À medida que mais sólidos dissolvidos são adicionados, a condutividade específica da água aumenta. Altos valores podem indicar características corrosivas da água. A condutividade representa uma medida indireta da concentração de poluentes (ALMEIDA e NASCIMENTO, 2011). 
Os resultados de turbidez mostram aumento significativo no período de estiagem, sendo que o ponto 1, expôs a maior variância sendo 36 NTU, o menor valor da variável ocorreu no mesmo período sendo esse de 2 NTU no ponto 3. No período de cheia o maior valor foi no ponto 1 com 24 NTU, os pontos 2 e 4 tiveram 15 NTU e os demais pontos ( 3 e 5) tiveram 14 NTU, explicado na Figura 5.

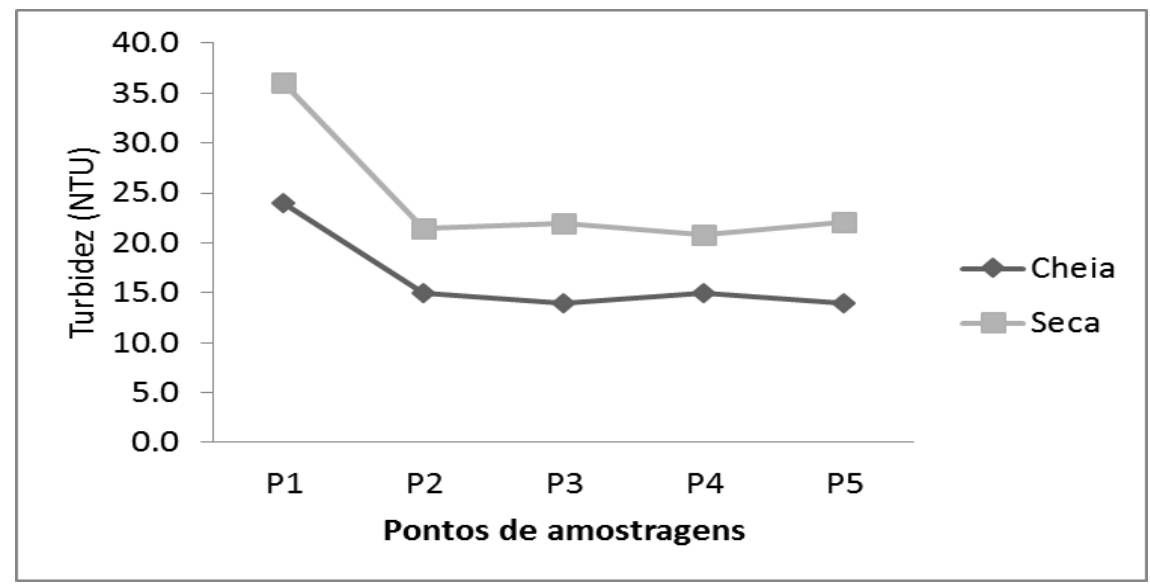

Figura 5: Comparação dos valores de turbidez, nos pontos de amostragens, nos períodos de Seca e Cheia na Lagoa Paiaguás em 2011.

A turbidez de água trata-se do grau de atenuação de intensidade que um feixe de luz sofre ao atravessá-la, onde esta redução dá-se por absorção e espalhamento, uma vez que as partículas que provocam turbidez nas águas são maiores que o comprimento de onda da luz branca devido à presença de sólidos em suspensão, tais como partículas inorgânicas (areia, silte, argila), de detritos orgânicos, algas, bactérias e plâncton em geral, entre outros. (SEMA, 2010).

A Demanda Bioquímica de Oxigênio é o parâmetro que representa a quantidade de oxigênio necessária para oxidar a matéria orgânica por decomposição microbiana aeróbica para uma forma inorgânica estável. Neste estudo considerou-se a quantidade de oxigênio consumido durante 5 dias com temperatura de incubação de 20C (SEMA, 2011). O oxigênio apresentou maior concentração no período da seca (Figura 6), estando os valores em todos os 5 pontos estáveis em $3 \mathrm{mg} \mathrm{O}^{2} / \mathrm{L}$, no período de cheia esteve com poucas variações de concentrações onde o menor valor foi no ponto $3 \mathrm{com} 1 \mathrm{mg} \mathrm{O} / \mathrm{L}$, os demais pontos tiveram $2 \mathrm{mg} \mathrm{O}^{2} / \mathrm{L}$.

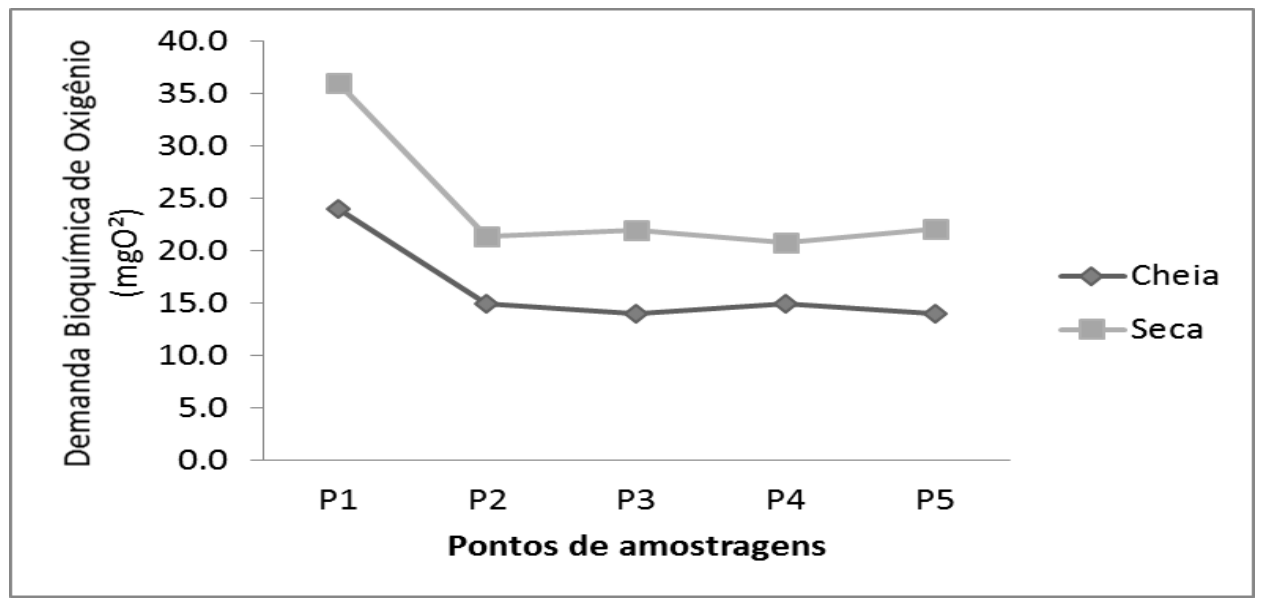

Figura 6: Comparação dos valores de Demanda Bioquímica de Oxigênio, nos pontos de amostragens, nos períodos de Seca e Cheia na Lagoa Paiaguás em 2011. 
Os maiores acréscimos desse parâmetro, num corpo d'água, geralmente são oriundos por despejos de origem orgânica. Os valores de oxigênio dissolvido (OD) da Lagoa Paiaguás foram contrários aos estudos de Silva et al., (2011) no Córrego Mangueirão em Aquidauana, MS, as maiores concentrações OD foram registradas no período chuvoso. Logo, na Lagoa Paiaguás esse parâmetro exposto na Figura 7 variou de 4,4 a 11,02 mg/L. Sendo os menores valores obtidos no período chuvoso, nos Pontos 1 e 5 (4,4mg/L e 5,84mg/L), respectivamente.

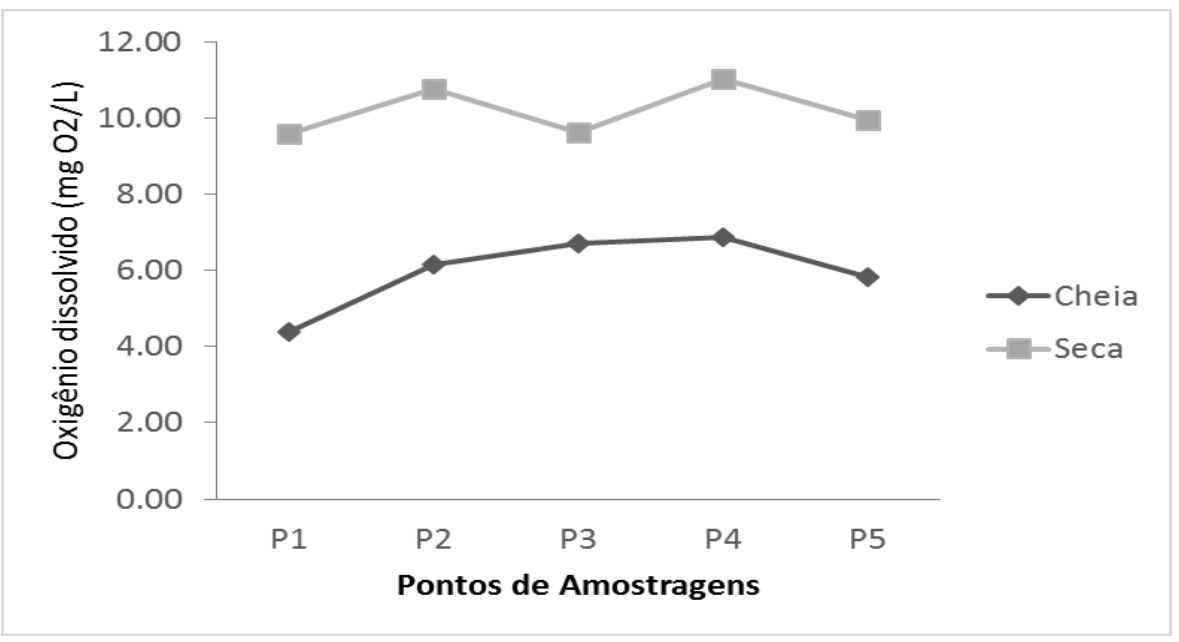

Figura 7: Comparação dos valores de oxigênio dissolvido, nos pontos de amostragens, nos períodos de Seca e Cheia na Lagoa Paiaguás em 2011.

O Ponto 4, referente a coleta do mês de agosto, ocorreu a maior quantidade de OD, $(11,02$ $\mathrm{mg} / \mathrm{L}$ ). Alongo uma maior distinção de espécies comparando com os outros pontos amostrais porém não se pode afirmar se chegou ao estado de eutrofização. No período de seca ocorreram os maiores valores de Nitrogênio Nitrato os pontos 1, 2 e 5 tiveram 0,30 (mg/L) e os pontos 3 e 4 tiveram $0,20(\mathrm{mg} / \mathrm{L})$. No período de cheia registrou-se a maior concentração no ponto 2 com 0,40 (mg/L), os pontos 1, 3 e 4 tiveram estabilidade de concentração com $0,20(\mathrm{mg} / \mathrm{L})$ descritos na Figura 9.

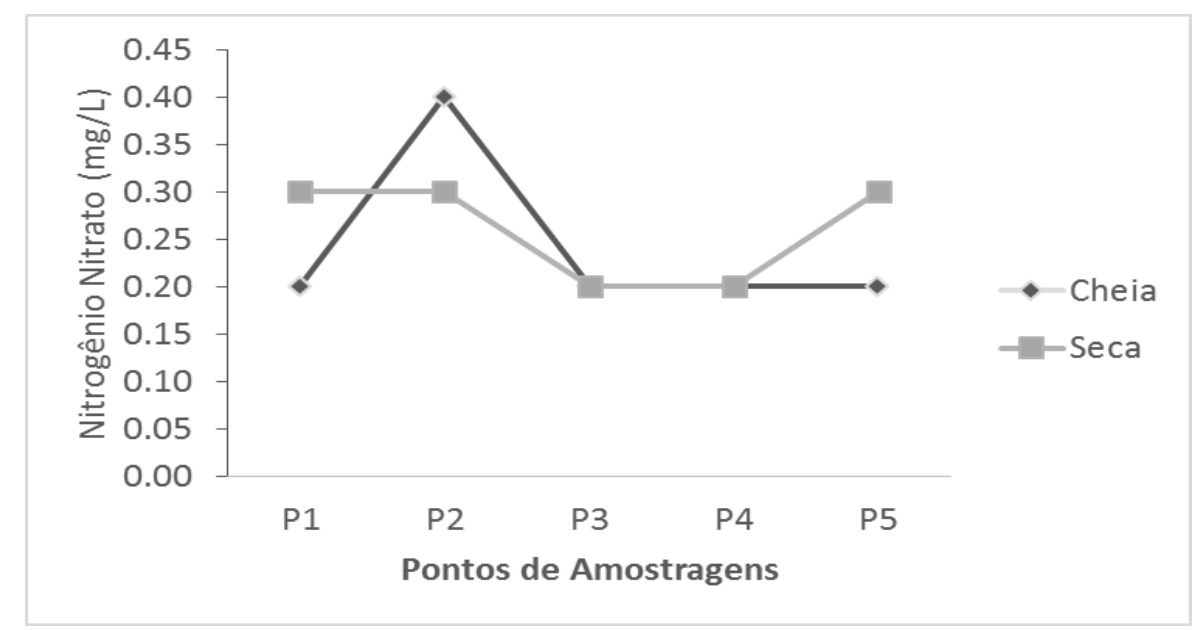

Figura 9: Comparação dos valores de Nitrogênio Nitrato, nos pontos de amostragens,

O Nitrogênio Nitrato é a principal forma de nitrogênio encontrado nas águas sua principal fonte são os dejetos humanos e de animais. Estimula o desenvolvimento de plantas, sendo que os 
organismos aquáticos, como as algas florescem na presença destes (SEMA, 2011). Nitrogênio Nitrato indica poluição antiga sendo ele produto da oxidação de nitrogênio nitrito sendo esse composto nitrogenado de grande estabilidade (BRAGA et al.,2009).

\section{CONCLUSÃO}

As variáveis limnológicas desempenham papel de diagnostico ambiental, mostrando através de seus resultados alterações ora naturais ou causadas por ações antrópicas. Os parâmetros expressão a influencia na profusão de microalgas Chlorophytas de forma direta ou indireta, pois em geral populações de micro-organismos possuem perfil de reprodução e sobrevivência indo de acordo com a qualidade do ambiente, desempenhando assim funções de bioindicadores ambientais, podendo vir a fornecer informações de causa e efeito dentro do ecossistema.

Embora as margens da Lagoa apresentaram mata ciliar com vegetação típica de cerrado e cerradão é plausível ressaltar a ocorrência de uma macrofita aquática exótica que pode vir a descaracterizar o ambiente, além da ocorrência de uso e ocupação do solo no entorno da Lagoa.

Através das informações nesse estudo geradas é oportuno que sejam tomadas medidas como o acompanhamento a longo prazo tanto da qualidade da água quanto das intervenções humanas, afim de verificar a dinâmica e o equilíbrio desse ecossistema. Além de serem dadas a progressão de pesquisas na área servindo como informações que possam gerar contribuições para a gestão dos recursos hídricos.

\section{REFERÊNCIAS BIBLIOGRÁFICAS}

ALMEIDA, E. M ; NASCIMENTO, O. C. (2011). Avaliação da Qualidade da Água da Microbacia do Córrego Pau Grosso Impactada por Exploração Garimpeira, Nova Marilândia- MT. - Utilizando o IQANSF. Monografia (bacharelado e licenciatura em Ciências Biológicas). Universidade de Várzea Grande, Mato Grosso.

ALVES, E. C. R. F; SILVINO, A.N.O; ANDRADE, N. L. R; SILVEIRA, A. (2009, Jul/Set). Gestão dos Recursos Hídricos no Estado de Mato Grosso. RBRH - Revista Brasileira de Recursos Hídricos v. 14 n.3 p.69-80.

APHA - American Public Health Association /AWWA - American Water Works Association \& WPCF/Water Pollution Control Federation. (2005). Standard Methods. Washington: Ed. APHA. $21^{\mathrm{a}} \mathrm{Ed}$.

BICUDO, C. E. M; BICUDO, R. M. T. (1970). Algas de águas continentais brasileiras. São Paulo: Fundec.

BICUDO,C.E.M; MENEZES, M. (2006). Gêneros de algas continentais do Brasil (chave para identificação e descrições). 2. ed. São Carlos: Rima.

BORTOLINI, J.C. et al. (2010 a). Chlorococcales planctônicas do Rio São João, Parque Nacional do Iguaçu Paraná, Brasil. Hoehnea . v. 37, n. 2, p. 315-330, 3 fig. 
BORTOLINI,J.C. et al. (2010 b). Cosmarium Corda ex Ralfs (Desmidiaceae) em um lago artificial urbano, Paraná, Brasil. Revista Brasileira de Biociências. Porto Alegre, v. 8, n.3, p.229-237.

BOURRELY, P. Les algues d' eau douce, Initiation à Systématique, I. Les Algues Vertes- Paris: Boubeé \& Cie. 1972.

BRAGA, J. K. et al. (2009). Análise dos Composto Nitrogenados de um Córrego com Influência de Lançamento de Esgoto Doméstico in natura em, Várzea Grande. In: Seminário Estadual de Recursos Hídricos em Mato Grosso, 9, Cuiabá, 14 a 16 de Out, Anais: Governo do Estado de Mato Grosso, Secretaria de Estado do Meio Ambiente- SEMA, Superintendência de Recursos Hídricos- SURH. p.129.

CALIJURI, M. C. (1988). Respostas fisioecológicas da comunidade fitoplanctônica e fatores ecológicos em ecossistemas com diferentes estágios de eutrofização. Tese (Doutorado em Engenharia) - Escola de Engenharia de São Carlos, Universidade de São Paulo, São Carlos, p. 293.

CETESB - Companhia de Tecnologia de Saneamento Ambiental. (1988). Coleta e Preservação de Amostras de Água. São Paulo.

CONAMA. Conselho Nacional de Meio Ambiente. (2005). Resolução 357 de 17 de Março de 2005. Dispões sobre a classificação dos corpos de água e diretrizes ambientais para o seu enquadramento, bem como estabelece as condições e padrões de lançamento de efluentes, e dá outras providências. Disponível em:< http://www.mma.gov.br/port/conama/res/res05/res35705.pdf./>. Acessado em 16 abr. 2012.

CUNHA, D.G.F; CALIJURI, M.C. (2010, Out/Dez.). Análise probabilística de ocorrência de incompatibilidade da qualidade da água com o enquadramento legal de sistemas aquáticos estudo de caso do rio Pariquera-Açu (SP). Eng Sanit Ambient. v.15, n.4, p. 337-346.

GENTIL, R.C. (2007). Estrutura da Comunidade fitoplanctônica de pesqueiros da Região Metropolitana de São Paulo, SP,em dois períodos: primavera e verão. Tese (Doutorado em Biodiversidade Vegetal e Meio Ambiente) - Instituto de Botânica da Secretaria de Meio Ambiente do Estado de São Paulo, São Paulo. p. 186 p.

HENTSCHKE, G. S; TORGAN, L.C. (2010). Chlorococcales lato sensu (Chlorophyceae, excl. Desmodesmus e Scenedesmus) em ambientes aquáticos na Planície Costeira do Rio Grande do Sul, Brasil. IHERINGIA. Sér.Bot. Porto Alegre, v. 65, n. 1, p. 87.

JUNQUEIRA, M. V; AMARANTE, M. C; DIAS, C. F. S; FRANÇA, E. S. (2000). Biomonitoramento da qualidade das águas da Bacia do Alto Rio das Velhas (MG/Brasil) através de macroinvertebrados. Acta Limnologica Brasiliensia, v.12, n.1, p. 73-87.

MARQUES, D. M. et al. (2002). Consolidação e Homogeneização de Procedimentos para Monitoramento e Avaliação da Qualidade da Água: Procedimentos vigentes na FEMA/MT: Procedimentos Básicos para Monitoramento e Avaliação da Qualidade de Água. Cuiabá: Fundação Estadual do Meio Ambiente. 
SEMA-MT-Secretaria de Estado do Meio Ambiente. (2004). Projeto: Monitoramento da Qualidade da Água da Lagoa Paiaguás. Cuiabá.

SEMA-MT. (2010). Relatório de Avaliação da Balneabilidade das Praias Fluviais- 2008 a 2010. Cuiabá.

SEMA-MT. (2007). Relatório de Biomonitoramento da Qualidade da Água na Lagoa Paiaguás, Cuiabá- MT em Março de 2007. Cuiabá.

SEMA-MT. (2011). Relatório de Monitoramento da Qualidade da Água da Região Hidrográfica do Paraguai 2007 a 2009. Cuiabá.

SILVA, A. V. C; SOUZA, G. R; ANDRADE, M. H. S. (2011). Aspectos Limnológicos do Córrego Mangueirão, em Aquidauana, MS. In: CONGRESSO DE BIÓLOGOS DO CRBio-01, 20, Corumbá, 17 a 20 jul. Anais: Conselho Regional de Biologia CRBio-01,2011, p. 142.

SOUZA, K.F; MELO, S. (2011). Levantamento Taxonômico de Desmidias (Chlorophyta) do lago Novo (Amapá, Brasil): Gêneros Staurastrum, Staurodesmus e Xanthidium. Acta Amazonica. v. 41, n. 3, p. 335-346.

AGRADECIMENTOS: Universidade Federal de Mato Grosso, Secretaria de Estado de Meio Ambiente de Mato Grosso e Universidade de Cuiabá. 